\title{
A Hospital Healthcare Monitoring System Using Wireless Sensor Networks
}

\author{
Media Aminian ${ }^{1}$ and Hamid Reza Naji ${ }^{2 *}$
}

${ }^{1}$ Department of Computer, Science and Research branch, Islamic Azad University, Kerman, Iran

${ }^{2}$ College of Electrical and Computer Engineering, Kerman Graduate University of Technology, Iran

\begin{abstract}
In a hospital health care monitoring system it is necessary to constantly monitor the patient's physiological parameters. For example a pregnant woman parameters such as blood pressure (BP) and heart rate of the woman and heart rate and movements of fetal to control their health condition. This paper presents a monitoring system that has the capability to monitor physiological parameters from multiple patient bodies. In the proposed system, a coordinator node has attached on patient body to collect all the signals from the wireless sensors and sends them to the base station. The attached sensors on patient's body form a wireless body sensor network (WBSN) and they are able to sense the heart rate, blood pressure and so on. This system can detect the abnormal conditions, issue an alarm to the patient and send a SMS/E-mail to the physician. Also, the proposed system consists of several wireless relay nodes which are responsible for relaying the data sent by the coordinator node and forward them to the base station. The main advantage of this system in comparison to previous systems is to reduce the energy consumption to prolong the network lifetime, speed up and extend the communication coverage to increase the freedom for enhance patient quality of life. We have developed this system in multi-patient architecture for hospital healthcare and compared it with the other existing networks based on multi-hop relay node in terms of coverage, energy consumption and speed.
\end{abstract}

Keywords: Patient; Blood pressure; Hospital healthcare; Wireless body sensor network; Energy consumption; End-to-end delay; Pregnant woman

\section{Introduction}

Body sensor network systems can help people by providing healthcare services such as medical monitoring, memory enhancement, medical data access, and communication with the healthcare provider in emergency situations through the SMS or GPRS $[1,2]$. Continuous health monitoring with wearable [3] or clothing-embedded transducers [4] and implantable body sensor networks [5] will increase detection of emergency conditions in at risk patients. Not only the patient, but also their families will benefit from these. Also, these systems provide useful methods to remotely acquire and monitor the physiological signals without the need of interruption of the patient's normal life, thus improving life quality $[6,7]$.

Although present systems allow continuous monitoring of patient vital signs, these systems require the sensors to be placed bedside monitors or PCs, and limit the patient to his bed. But now, there is no relation between the sensors and the bedside equipment due to the wireless devices and wireless networks [6]. These systems do not require the patient to be limited to his bed and allow him to move around but requires being within a specific distance from the bedside monitor. Out of this range, it is not possible to collect data. In most cases, health monitoring will be done by infrastructure-oriented wireless networks such as commercial cellular/3G networks or wireless LANs. But, the coverage of the infrastructure-oriented networks changes with time or location. Sometimes, the coverage of wireless network is not available, or the coverage is available but we cannot access to the network due to a lack of available bandwidth. So, with these problems and restrictions, continuous health monitoring is not possible and emergency signals may not be transmitted from a patient to healthcare providers. Under these conditions, we can reach to continuous health monitoring by using ad hoc wireless networks that can transmit vital signs over a short-range [8,9]. Lai et al. [10] presented a WMHRN for reliable and real-time transmission. In most systems the health data from multiple patients can be relayed wirelessly using multi-hop routing scheme to a base-station in [11-13].
In an example of the application of this system is controlling a pregnant woman. A pregnant woman's blood pressure should be the same as any other person's normal blood pressure. It is important to monitor the blood pressure during pregnancy, to watch for preeclampsia. These women need frequent BP checks. If BP goes too high, the patient may be hospitalized. But, the patient is limited to her bed in hospital.

In the proposed system, the patient's physiological signals are acquired by the sensors attached on the patient body, and are then transmitted to the remote base-station and also a PC for storing and analyzing. According to table 1 [14], in indoor environments, the signal strength of access points can be weakened by $30-90 \%$ as it passes through the obstructions (for example, when the two wireless devices or relay nodes are in different rooms, and the door which connects the rooms are closed). With the increasing number of obstructions between the nodes, we can observe more packet loss and more dead spot that

\begin{tabular}{|l|l|}
\hline Distance- obstructions & Packet lost rate \\
\hline $3 \mathrm{~m}$ & $0.13 \%$ \\
\hline $5 \mathrm{~m}$ & $0.19 \%$ \\
\hline $5 \mathrm{~m}-1$ door & $0.32 \%$ \\
\hline $10 \mathrm{~m}$ & $0.85 \%$ \\
\hline $10 \mathrm{~m}-1$ door & $1.08 \%$ \\
\hline $10 \mathrm{~m}-2$ doors & $1.23 \%$ \\
\hline Moving patient & $20.15 \%$ \\
\hline
\end{tabular}

Table 1: Loss rate values for the in-house patient [14].

${ }^{*}$ Corresponding author: Hamid Reza Naji, College of Electrical and Computer Engineering, Kerman Graduate University of Technology, Iran, E-mail: hamidnaji@ieee.org

Received November 26, 2012; Accepted January 30, 2013; Published February 04, 2013

Citation: Aminian M, Naji HR (2013) A Hospital Healthcare Monitoring System Using Wireless Sensor Networks. J Health Med Inform 4: 121. doi:10.4172/21577420.1000121

Copyright: (c) 2013 Aminian M, et al. This is an open-access article distributed under the terms of the Creative Commons Attribution License, which permits unrestricted use, distribution, and reproduction in any medium, provided the original author and source are credited. 
Citation: Aminian M, Naji HR (2013) A Hospital Healthcare Monitoring System Using Wireless Sensor Networks. J Health Med Inform 4: 121. doi:10.4172/2157-7420.1000121

will cause a communication disconnection between the patient and the network. So, we have to increase the number of relay nodes within the indoor environments to cover the whole environment, and enhance the reliability for wireless connectivity. In addition, an emergency alert service using short message service (SMS) messaging is also added to the proposed system for emergency responses and rescues.

\section{Multi-Patient Body Sensor Network System Design}

In this paper, a ubiquitous healthcare prototype system for hospitals is designed. The concept of Ubiquitous healthcare system is to place unobtrusive wireless sensors on a person's body to form a wireless network which can communicate the patient's health status with base station connected to the monitoring PC.

The architecture and application of the proposed system are in figure 1. The system consists of four parts: (1) the WBSN includes four sensors which are responsible for collecting the physiological signals from patient, (2) the WMHRN(Wireless Multi-Hop Relay Node), consist of a number of wireless relay nodes which is in charge of forwarding the health data to the base station, (3) a BS (Base Station) which receives the relayed data and sends it to the PC through a cable and (4) and the graphical user interface (GUI) which is responsible for storing, analyzing and presenting the received data in graphical and text format, and sending an SMS to the healthcare provider or patient's family in emergency conditions through the GPRS or GSM modem.

\section{Coordinator and sensors nodes}

This section describes the components that make up the WBSN and their functionality.

Sensor nodes: Each node in the network has a different role. All the sensors are wireless and sense different physiological parameters in a given interval and simultaneously, the sampling interval is determined by the physician. For example sensors for pregnant woman can be:

1) Motion Detection-Accelerometers: To measure blood pressure, the patient should be seated or lying [15]. So, these sensors can detect patient's position. If the patient is placed in a proper position, it will allow the other sensors to sense.

2) Blood Pressure and Heart Rate: These sensors can return blood pressure and heart rate.

3) Heart rate and Movement of the fetal: Detection of the heart rate and the movement of the fetal used by medical practitioners to assess the health of the fetal.

Coordinator Node: The coordinator node is a wireless node in the WBSN which is in charge of collecting and packaging the arrival signals from the other sensors, and send them to the base station. This node attaches on the patient's body and it works with battery. Each

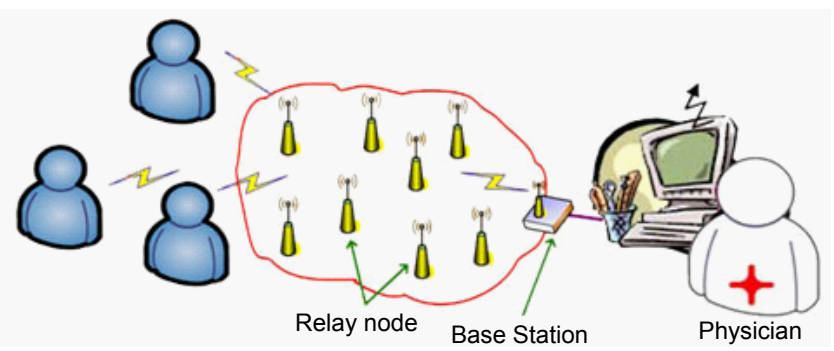

Figure 1: Architecture for proposed healthcare system in hospital. coordinator node is identified by a unique ID which is used to identify each patient in the network.

GPS: GPS is a space-based satellite navigation system that provides location information about the patient in the hospital which helps to hospital stuffs to finding the patients in emergency conditions. It is always carried by the patient. For example, Fahim et al. [16] presented a smart house to assists elderly people for independent living in their own homes. It facilitates the care giver assistant by tracking the elderly persons in their own homes and avoids certain accidents.

\section{The WBSN design}

As we mentioned to measure blood pressure, the patient should be seated or lying. So, these sensors can detect patient's position. If the patient is placed in a proper position, the motion sensors by sending a SENSE_START packet will allow the other sensors to sense. To reduce energy consumption, all the sensors wake up simultaneously and wait for a specified time until they receive the SENSE_START packet. If they receive it, they will send their readings to the coordinator node and then turn off their radio. We can observe the operation state diagram of the Blood Pressure (BP) sensor in figure 2. If each sample of data (such as the heart beat, BP etc.) is immediately encapsulated into a frame and transmits, the overhead will be increased and will cause excessive energy consumption. Therefore to decrease the overhead, we have used the burst transmission mechanism [10]. As shown in figure 3 , woman heart rate, woman blood pressure, fetal heart rate and fetal movement encapsulate in one frame. In this way, we achieve more energy conservation.

Among various existing MAC protocols, we have used Time division Multiple Access (TDMA) protocol for media access because there is a constant number of nodes between sensors and the coordinator node. TDMA divides the channel into individual time slots, which are then grouped into frames. In each time slot, only one node can transmit. TDMA is intrinsically energy-efficient. A sensor node can turn off its radio during all time slots in a frame. Moreover, when the number of nodes in a cluster changes, it is difficult for a TDMA protocol to dynamically change its frame length and time slot assignment [17]. The assigned time slot to the sensor nodes in the proposed WBSN has shown in figure 4. Also, to control data transmission and reduce collisions between data sent, we have used CSMA/CA (Carrier sense

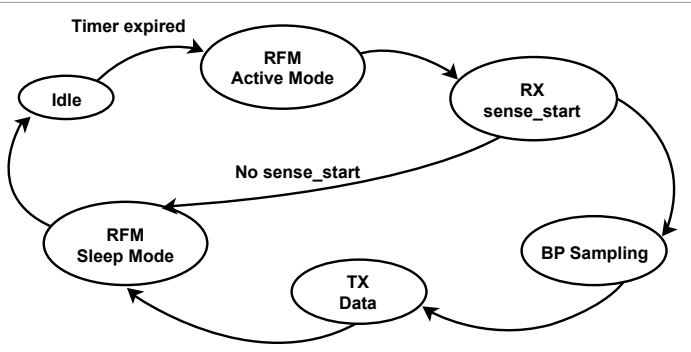

Figure 2: State transmission diagram of the BP sensor.

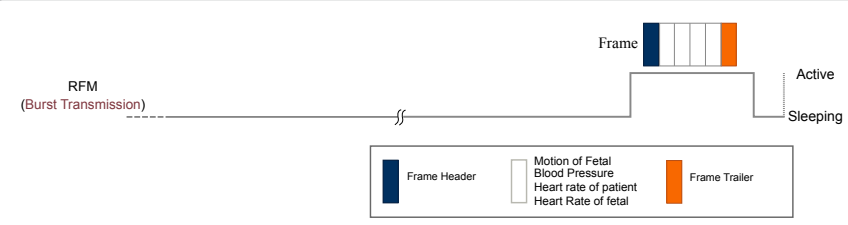

Figure 3: Burst transmission. 


\section{FRAME}

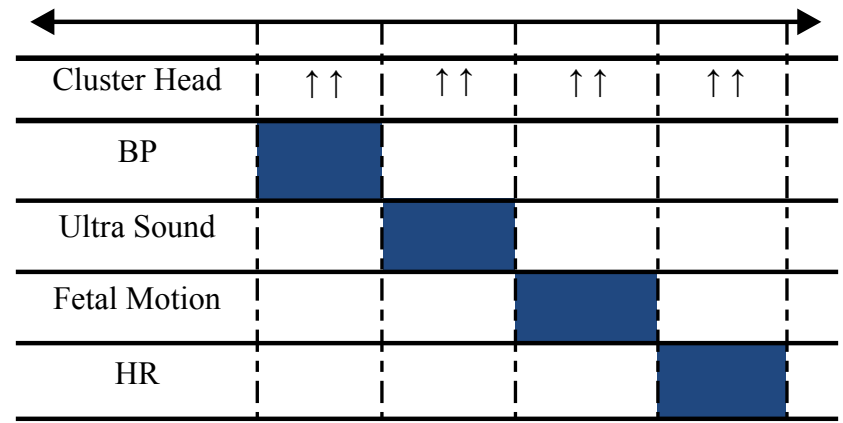

Figure 4: Time slots allocation to the sensors.

After receiving an FMP packet checks and turns on the timer If (current hop count $>$ previous hop count) Drop the packet

Else If (current hop count $<$ previous hop count)

Keep it in routing table.

Increase the current hop count by 1 .

Add the ID to the Next Hop

And send it to the neighbors.

Else IF( the timer is expired) send an FMP_END to the BS

Figure 5: The proposed algorithm to find the minimum path.

multiple access with collision avoidance) MAC. In this design we used the RTS/CTS (Ready to Send/Clear to send). When a coordinator node wants to send data to a relay node first it will check the transmission channel. If the channel is free then the relay node will send a CTS packet to the coordinator node. This simple MAC protocol allows multiple coordinator nodes to reliably transmit their data to a relay node without any significant delay.

\section{The WMHRN design}

The WMHRN consists of several wireless nodes. Relay node has only routing capability to transfer physiological signals, such as heart beat, BP, and other biomedical signals to the base station or to surrounding other relay nodes. As compared with the coordinator node, the power can be supplied from the battery or the AC adapter. Since hospitals have fluorescent lights in the hallways that are always on, it is advantageous to operate these relay nodes by scavenging light energy. This would result in a huge cost savings over time (this arrangement eliminates the need to monitor and replace batteries).

The operation of the WMHRN of our proposed system can be divided into two phases. The first phase is finding the minimum path from the coordinator node to the base station. The second phase is the data transmission stage through the minimum path. By these nodes and the first phase, the end-to-end delay will be decreased. "Endto-End" delay refers to the time taken for a packet to be transmitted across a network from source to destination. By reducing the end-toend delay, the speed will be increased. When the speed is high, system can detect abnormal physiological parameters and issue alarm rapidly. Also, several nodes in an indoor environment can reduce the dead spots and extend the patient's freedom. Therefore, patient signals can be accessed anywhere of hospital without any disconnection between patient and the network and any limitation for patient movement.

To find the minimum path, we have proposed an algorithm that it has shown in figure 5. The first phase begins with sending a FMP (Find Minimum Path) packet from the BS. FMP consists of <hop count, source, node ID $>$ to find minimum path. When a node receives an FMP, it checks the hop count. If the hop count is smaller than hop count in the previous FMP, it will record the packet to its route table. Then the node adds its ID to the "node ID" field and then sends it to its neighbors. After a specified time, the minimum path from nodes to BS will be found and each node returns an FMP_END packet which is similar to FMP and then sends the packets to the BS through the path in their route table. When a node receives an FMP_END, it records the ID in its route table. So, the minimum path from the BS to nodes will be determined.

The second phase starts with broadcasting a TRANS_MODE packet in the network from the BS. When the coordinator node receives this packet, it can transmit the data packet into the network.

\section{Design of GUI and monitoring system}

The installed software in the remote computer receives real-time patient's data from the base station and processes them to detect anomalies. The GUI allows users to see history of medical information and current vital signs in real-time. Table 2 shows a partial list of physiological conditions that cause alerts. Figure 6 shows an alert in the software application when the BP is out of the threshold. By detection an alert, a SMS/E-mail will be sent to the patient's family or the healthcare provider through the GSM modem/GPRS.

A GSM modem is a specialized type of modem which accepts a SIM

\begin{tabular}{|l|l|}
\hline Alert Type & Detection Parameter \\
\hline Tachycardia of woman & $>100 \mathrm{bpm}$ \\
\hline Bradycardia of woman & $<80 \mathrm{bpm}$ \\
\hline Tachycardia of fetal & $>110 \mathrm{bpm}$ \\
\hline Bradycardia of fetal & $<100$ \\
\hline BP of woman & $>139 / 90$ (systolic/diastolic) \\
\hline movements & From past 4 readings $>=3$ \\
\hline Moving patient & $20.15 \%$ \\
\hline
\end{tabular}

Table 2: Alert detection parameters [13].

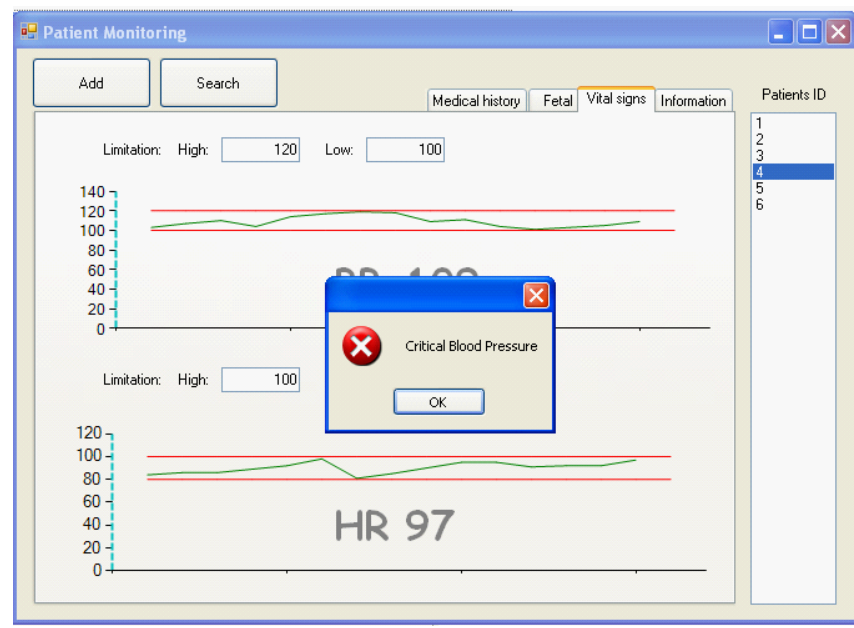

Figure 6: An alert when the patient's blood pressure level is critically low. 
Citation: Aminian M, Naji HR (2013) A Hospital Healthcare Monitoring System Using Wireless Sensor Networks. J Health Med Inform 4: 121. doi:10.4172/2157-7420.1000121

Page 4 of 6

card, and operates over a subscription to a mobile operator, just like a mobile phone. From the mobile operator perspective, a GSM modem looks just like a mobile phone. When a GSM modem is connected to a computer, this allows the computer to use the GSM modem to communicate over the mobile network. While these GSM modems are most frequently used to provide mobile internet connectivity, many of them can also be used for sending and receiving SMS and MMS messages.

\section{Performance evaluation and discussion}

The previous section gave a comprehensive schema of our proposed WBSN. One way to analyze the performance of WBSN system is to measure the end-to-end delay, energy consumption, coverage range. So, we have simulated the proposed system in OMNet++ and the results are shown and explained as follows.

\section{Coverage range}

Due to use of several relay nodes in the environment, it is not required to use high frequency band. So, we can transmit the data through a short-range frequency module (RFM) inside the coordinator node and wireless relay nodes with minimum energy consumption. There are medical bands such as MICS (Medical Implant Communication Service) with 10 meters coverage and $402-405 \mathrm{MHz}$ frequency band that could be used for body-area network applications because of its low transmitter power [18]. According to figure $\mathrm{X}$, to cover a hospital with $50 \mathrm{~m} \times 20 \mathrm{~m}$ dimensions without any obstructions in it such as doors and walls, we need 7 relay nodes. Therefore, there is not any dead spot in this environment. Whereas, with several obstructions on the way of signals to prevent of weakening signal and loss signals we need more relay nodes (Figure 7).

\section{Energy consumption}

There is a relation between energy consumption and lifetime of the network. A challenge of WBSN is patient quality of life during the monitoring. So, we have provided the quality by reducing energy consumption in coordinator node. Coordinator node has an important role in the network such as aggregation of sensory data, data packaging and transmitting them to the base station. Therefore, we have to prolong the lifetime of coordinator node. If the lifetime be short, the patient has to recharge the battery of coordinator node in short intervals, and if the patient forget to recharge the battery, it may be dangerous for the patient.

If each sample of data by the sensors is immediately encapsulated into a frame and then transmitted, the energy consumption will be increased. Therefore, the burst transmission mechanism is employed.

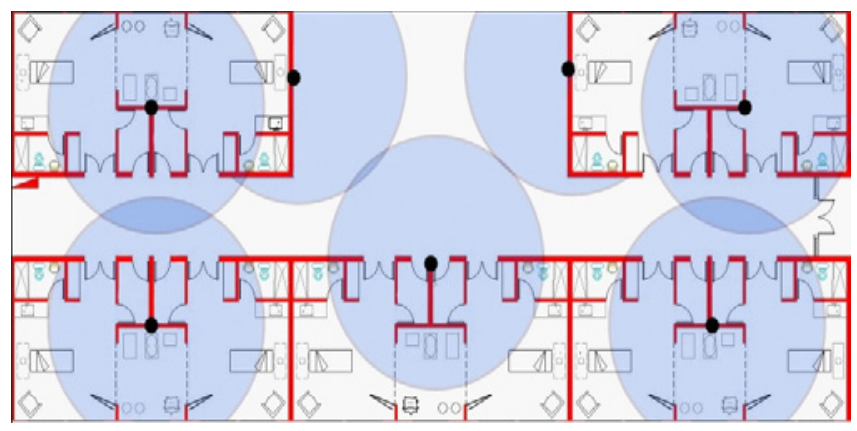

Figure 7: A hospital with 7 relay nodes for coverage of the whole environment.
We can observe the relation between energy consumption and number of samples in a packet in figure 8 .

Assuming that the coordinator node's battery is $5000 \mathrm{~mJ}$ and the interval of data transmission is once every three hours. In normal case, the battery can continue to work for three days. But, in burst transmission, the battery lifetime will be upgraded to 9 days.

\section{End-to-end delay}

When the delay is high, it seems the speed is low. Because of this, unusual signs are diagnosed late and this could be very dangerous for the patients with blood pressure. Due to the crucial application, it needs an acknowledgement (ACK) for each data packet. There are two scenarios for ACK delivery. In the first scenario, when the BS receives a data packet, it will return an ACK to the coordinator node. In the second scenario, when each intermediate node receives the data packet, it will return an ACK to the sender. Therefore, end-to-end delay and energy consumption in coordinator node will be reduced in the second scenario. Figure 9 presents the relation between distance (no. of hops) and the end-to-end delay in two scenarios of ACK delivery. On the other hand, the relation between distance (no. of hops) and the end-toend delay when a packet gets lost in the way and the coordinator node has to retransmit the data packet is shown in figure 10.

\section{Comparison of WBSN systems}

This section discusses and compares the existing WBSN systems based on multi-hop methodology. Yuce [17] presents a multi-hopping network for a multi-patient system that can be used in medical environments for remote monitoring of physiological parameters. But the coverage range by this system is 10 meters, and the patients cannot move around freely. Chung et al. [11] designed a multi-patient network

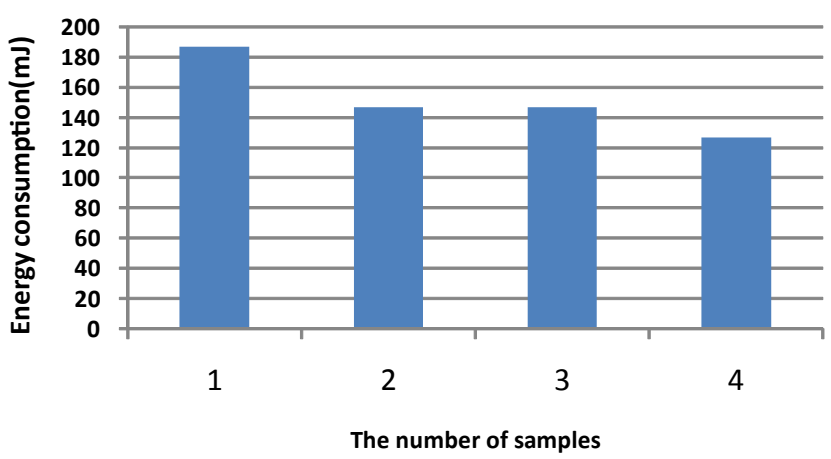

Figure 8: The relation between energy consumption and number of samples.

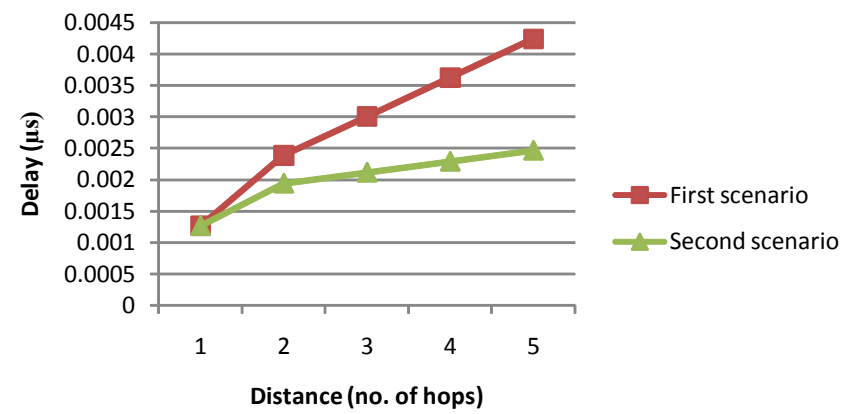

Figure 9: The relation between distance (no. of hops) and the end-to-end delay. 
Citation: Aminian M, Naji HR (2013) A Hospital Healthcare Monitoring System Using Wireless Sensor Networks. J Health Med Inform 4: 121. doi:10.4172/2157-7420.1000121

Page 5 of 6

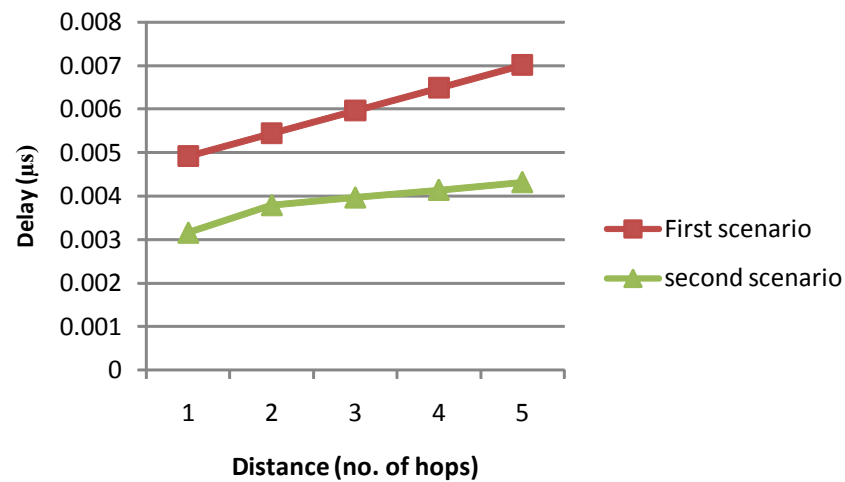

Figure 10: End-to-end delay based on retransmission of data packets.

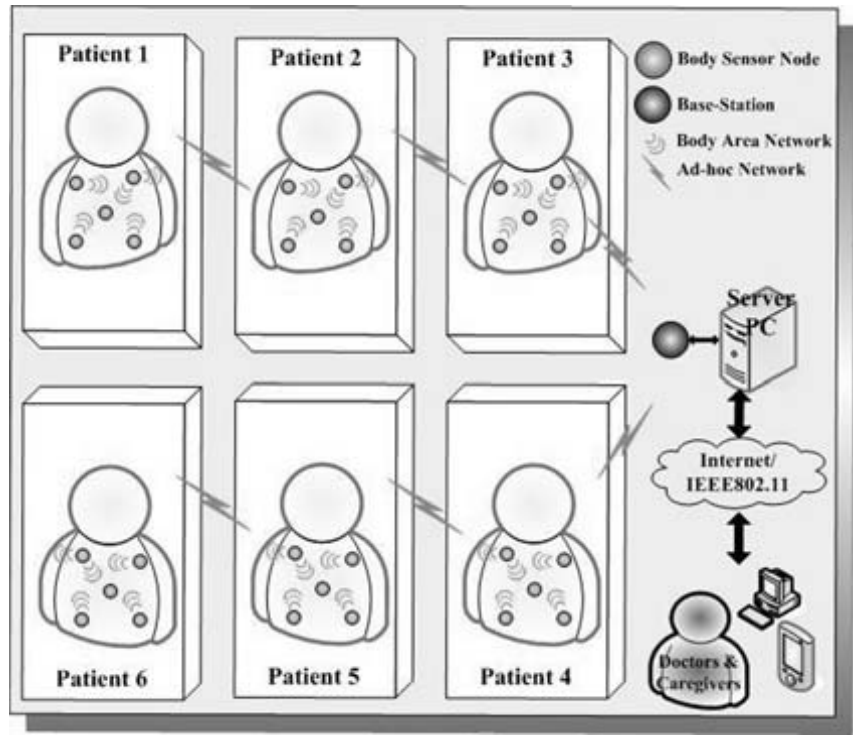

Figure 11: The presented architecture [10]

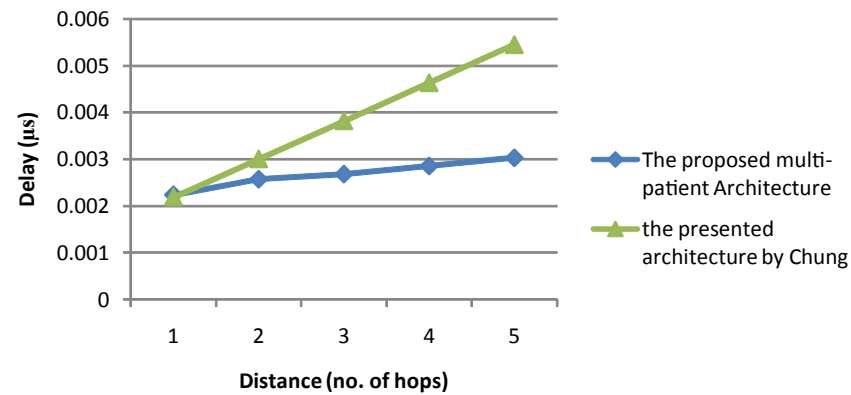

Figure 12: Comparison between the proposed architecture and the presented architecture by Chung [11] in term of delay.

using multi-hop routing scheme for transmission of data. We can see the architecture in figure 11. It seems to be a good idea because the additional devices between the patient and BS have removed and it can reduce the cost. But, according to figure 12, the end-to-end delay [11] is more than the proposed system in this paper, and the energy consumption has increased at the intermediate nodes and they need to be recharged more. Figure 13 has shown the energy consumption at the

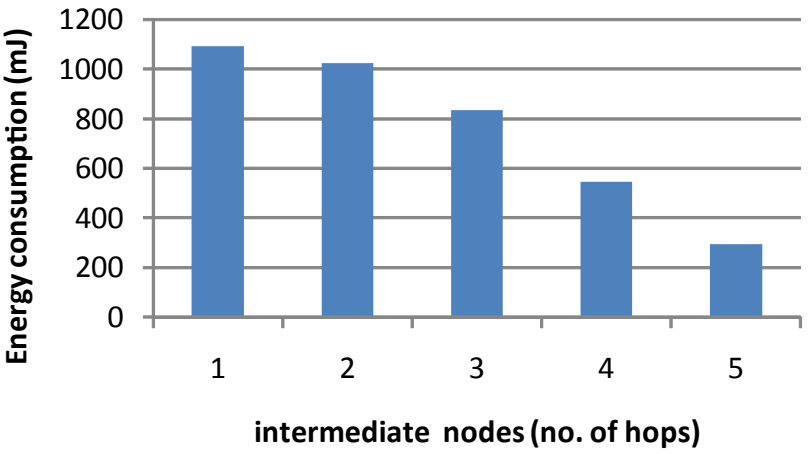

Figure 13: Energy consumption for one transmission at the intermediate nodes.

intermediate nodes. Energy consumption at the closer nodes to base station is more than the other nodes. Another disadvantage is that the patient is dominated by signals and it is harmful for body of patients in the long time.

\section{Conclusion}

Wireless BSN technology is emerging as a significant element of next generation healthcare services. In this paper we proposed a mobile physiological monitoring system, which is able to continuously monitor the patient's heart beat, blood pressure and other critical parameters in the hospital. The entire system consists of a coordinator node to acquire the patient's physiological data, a WMHRN to forward the data and a BS to collect the data. The system is able to carry out a long-tem monitoring on patient's condition and is equipped with an emergency rescue mechanism using SMS/E-mail.

Also, we have simulated the proposed WBSN in OMNet++ simulator and have compared this with existing WBSN systems in terms of coverage, energy consumption and delay time and we have observed the proposed system has better performance than others existing WBSN systems.

\section{References}

1. Stanford V (2002) Using pervasive computing to deliver elder care. IEEE Pervasive Computing 1: 10-13.

2. Mcfadden T, Indulska J (2004) Context-aware environments for independent living, In Proceedings of the 3rd National Conference of Emerging Researchers in Ageing, Brisbane, Australia.

3. Anliker U, Ward JA, Lukowicz P, Troster G, Dolveck F, et al. (2004) AMON: wearable multi-parameter medical monitoring and alert system. IEEE Trans Inf Technol Biomed 8: 415-427.

4. Cho G, Yoo1 SK (2009) Wearable ECG Monitoring System Using Conductive Fabrics and Active Electrodes, Proceedings of the 13th International Conference on Human-Computer Interaction, Berlin, Heidelberg.

5. Darwish A, Hassanien AE (2012) Wearable and Implantable Wireless Sensor Network Solutions for Healthcare Monitoring. Sensors 12: 12375-12376.

6. Shnayder V, Chen B, Lorincz K, FulfordJones TRF, Welsh M (2005) Senso Networks for Medical Care, Proceedings of the 3rd international conference on Embedded networked sensor systems, New York, USA.

7. Alemdar H, Ersoy C (2010) Wireless sensor networks for healthcare: A survey Computer Networks 54: 2688-2710.

8. Varshney U (2005) Using wireless networks for enhanced monitoring of patients. International Journal of HealthCare Technology and Management 6: 489-499.

9. Varshney U (2003) Pervasive healthcare. IEEE Computer 12: 138-140. 
Citation: Aminian M, Naji HR (2013) A Hospital Healthcare Monitoring System Using Wireless Sensor Networks. J Health Med Inform 4: 121. doi:10.4172/2157-7420.1000121

Page 6 of 6

10. Lai CC, Lee RG, Hsiao CC, Liu HS, Chen CC (2009) A H-QoS-demand personalized home physiological monitoring system over a wireless multi-hop relay network for mobile home healthcare applications. Journal of Network and Computer Applications 32: 1229-1241.

11. Chung WY, Walia G, Lee YD, Myllyla R (2007) Design Issues and implementation of Query-Driven Healthcare System Using Wireless Sensor Ad-hoc Network. IFMBE Proceedings 13: 99-104.

12. Hande A, Polk T, Walker W, Bhatia D (2006) Self-Powered Wireless Sensor Networks for Remote Patient Monitoring in Hospitals. Sensors 6: 1102-1117.

13. Chung WY, Mo An S, Lee SC (2012) Real Time Multi-hop Routing Protocol for Healthcare System Based on WSN, 14th International Meeting on Chemical Sensors, Nuremberg, Germany.

14. Kyriacou E, Pattichis C, Hoplaros D, Kounoudes A, Milis M, et al. (2010) A system for Monitoring Children with Suspected Cardiac Arrhythmias Technical Optimizations and Evaluation. IFMBE Proceedings 29: 924-927.

15. Lowe SA, Brown MA, Dekker G, Gatt S, McLintock C, et al. (2008) "Guidelines for the Management of Hypertensive Disoders of Pregnancy". Society of Obstetric medicine of Australia and New Zealand.

16. Fahim M, Fatima I, Lee S, Lee YK (2012) Daily life activity tracking application for smart homes using android smartphone. Proceedings of 14th international conference on Advances Communication Technology (ICACT), Pyeong Chang

17. Yuce MR (2010) Implementation of wireless body area networks for healthcare systems. Sensors and Actuators A: Physical 162: 116-129.

18. Keong HC, Yuce MR (2008) Low data rate ultra wideband ECG monitoring system. Proceedings of IEEE Engineering in Medicine and Biology Society Conference, University of Newcastle, Callaghan, Australia. 